The following text is provided by DuEPublico, the central repository of the University DuisburgEssen.

This version of the e-publication released on DuEPublico may differ from a potential published print or online version.

Jiang, Wei; Kaiser, Thomas; Vinck, Han A. J.:

A Robust Opportunistic Relaying Strategy for Cooperative Wireless Communications

DOI: http://dx.doi.org/10.1109/TWC.2015.2506574

URN: urn:nbn:de:hbz:464-20170329-100458-1

Link: http://duepublico.uni-duisburg-essen.de/servlets/DocumentServlet?id=43612

Legal notice:

() ( 2015 IEEE. Personal use of this material is permitted. Permission from IEEE must be obtained for all other uses, in any current or future media, including reprinting/republishing this material for advertising or promotional purposes, creating new collective works, for resale or redistribution to servers or lists, or reuse of any copyrighted component of this work in other works.

Source: This is the author's version of an article that has been published in the journal IEEE Transactions on wireless communications. Changes were made to this version by the publisher prior to publication. The final version of record is available at http://dx.doi.org/10.1109/TWC.2015.2506574 


\title{
A Robust Opportunistic Relaying Strategy for Cooperative Wireless Communications
}

\author{
Wei Jiang, Member, IEEE, Thomas Kaiser, Senior Member, IEEE, and A. J. Han Vinck, Fellow, IEEE
}

\begin{abstract}
Using outdated channel state information(CSI) in an opportunistic relaying system (ORS) leads to wrong selection of the best relay, which substantially deteriorates its performance. In this article, therefore, we propose a robust cooperative scheme coined opportunistic space-time coding (OSTC) to deal with the outdated CSI. A predefined number (i.e., $N$ ) of relays, instead of a single relay in ORS, are opportunistically selected from $K$ cooperating relays. At the selected relays, $N$-dimensional orthogonal space-time coding is employed to encode the regenerated signals in a distributed manner. Then, $N$ branches coded signals are simultaneously transmitted from the relays to the destination, followed by a simple maximum-likelihood decoding at the receiver. To evaluate its performance, the closedform expressions of outage probability and ergodic capacity are derived, together with an asymptotic analysis that clarifies the achievable diversity. Analytical and numerical results reveal that a full diversity of $K$ is reaped by the proposed scheme when the knowledge of CSI is perfect. In the presence of outdated CSI, where the diversity of ORS degrades to one, the diversity of $N$ can be kept. Moreover, OSTC's capacity is remarkably higher than that of the existing schemes based on orthogonal transmission. From the perspective of multiplexing-diversity trade-off, the proposed scheme is the best cooperative solution until now.
\end{abstract}

Index Terms-Alamouti, cooperative diversity, decode-andforward, generalized selection combining, opportunistic relaying, outdated channel state information (CSI), space-time coding.

\section{INTRODUCTION}

In radio channels [1], the multi-path fading due to constructive and destructive interferences of received signals is a severe impairment. It is challenging for a receiver to correctly detect a signal without some form of diversity. Since time and frequency resources in a wireless system are extremely scarce, a particularly appealing approach is the utilization of antenna arrays, such as multiple-input multiple-output (MIMO) and massive MIMO [2], which can achieve higher diversity by simply installing additional antennas. Due to the limitations of cost, power supply and hardware size at carrier frequencies below $6 \mathrm{GHz}$ [3], it is impractical to exploit spatial diversity for mobile terminals in a cellular system or wireless nodes

Manuscript received May 03, 2015; revised August 05, 2015 and November 03, 2015; accepted November 26, 2015. This work was supported by the European Commission under H2020 5G-PPP COHERENT project (Grant No. 671639) and FP7 ABSOLUTE project (Grant No. 318632). This work was partly presented at the IEEE International Conference on Communications (ICC'14), Sydney, Australia, June 2014.

W. Jiang was with the institute of Digital Signal Processing, University of Duisburg-Essen, Duisburg, Germany. He is now with German Research Centre for Artificial Intelligence (DFKI GmbH), Kaiserslautern, Germany (email: wei.jiang@dfki.de).

T. Kaiser and A. J. H. Vinck are with the institute of Digital Signal Processing, University of Duisburg-Essen, Germany (e-mail: \{thomas.kaiser, han.vinck\}@uni-due.de). in ad hoc network. Hence, the cooperative communication [4] has been proposed to alleviate these limitation by exploiting the broadcast nature of radio signals in a relay channel [5], where multiple nodes can form a virtual array to cooperatively transmit their signals. Concretely, when a node sends a signal to its destination (e.g., a base station or wireless gateway), those neighboring nodes who overhear this signal could decode and retransmit. By combining multiple copied versions of the original signal at the receiver, an inherent spatial diversity, referred to as cooperative diversity [6], can be reaped.

Nowadays, it is practically impossible for mobile terminals or wireless nodes to transmit and receive signals simultaneously at the same frequency. The relays need to operate in a halfduplex mode where time- or frequency-division multiplexing is applied. Without loss of generality, we assume that signal transmission happens in two phases [7]. In the broadcast phase, the source transmits its signal in the source-relay channels while all relays listen. In the relaying phase, the relays decode and retransmit this signal in the relay-destination channels. However, a scheduling problem occurs: which relays should be selected and how to transmit the regenerated signals by the selected relays? In the literatures, several cooperative methods have been studied. Generalized selection combining (GSC) [8], which chooses multiple relays to orthogonally retransmit the original signal, suffers from a substantial loss of spectral efficiency. To avoid this penalty, the distributed beamforming [9] based on simultaneous transmission has been taken into account. Given a priori knowledge of forward channels, the relays could adjust the phases of their transmit signals for coherently combining at the receiver. The beamforming is very sensitive to phase noise. That is why a co-located antenna array must apply an antenna calibration to align phase distortions on different radio-frequency (RF) chains. However, an RF-chain calibration among spatially-distributed relays is impossible to implement. In [10], an approach called distributed space-time coding (DSTC) has been proposed. Although a full diversity can be achieved, designing such a code is infeasible since the number of distributed antennas is unknown and randomly varying. Moreover, the synchronization among simultaneously transmitting relays becomes challenging when the number of relays is large. In a nutshell, the aforementioned multi-relay selection methods are hard to be applied for practical systems.

In contrast, a single-relay approach referred to as opportunistic relaying system(ORS) [11] has been extensively verified as a simple but efficient way. Although $a$ single node with the best channel (in accordance to a given selection criterium) serves as a relay, its performance is as same as that of DSTC, which uses an all-participating strategy [12]. From the 
viewpoint of multiplexing-diversity trade-off, ORS provides no performance loss compared to DSTC, while avoiding complex implementations of the multi-relay selection methods.

Owing to the channel fading and feedback delay, the channel state information(CSI) at the time instant of relay selection may substantially differ from the CSI at the instant of using the selected relay to retransmit. The imperfect CSI imposes a possibility of wrong relay selection on ORS, which drastically deteriorates its performance. The impact of the outdated CSI on the performance of opportunistic relaying has been analyzed in the previous works [13]-[21]. Vicario et al. derived a closed-form expression of outage probability for decode-and-forward(DF) ORS [13]. Seyfi et al. investigated the impact of feedback delay and channel estimation error [14]. Kim et al. [15] evaluated the performance degradation with respect to symbol error probabilities. In the field of amplify-and-forward (AF) ORS, Torabi et al. presented a lot of results through [16]-[18]. The impact of the outdated CSI on partial relay selection has also been reported in [19], [20]. The error probabilities of ORS considering channel estimation errors have been derived in [21]. In summary, the following conclusions can be drawn: 1) The ORS is very vulnerable to the outdated CSI, where its achieved diversity is limited to one. 2) Regardless of how many relays cooperate, there is no diversity, even if the correlation coefficient of the actual and outdated CSI tends to one $(\rho \rightarrow 1)$. From a practical point of view, therefore, it is worth designing a robust cooperative strategy to combat the outdated CSI.

To the best knowledge of the authors, only a few proposals to deal with this problem have appeared until now. A method employing geolocation information has been proposed in [22]. But it makes sense only in a fixed wireless system rather than a mobile network. A strategy taking into account the knowledge of channel statistics has been given in [23]. Regardless of a remarkable increase of complexity, this scheme only achieves a marginal performance gain and its diversity is limited to one. Generalized selection combining [8] and its enhanced version called $N$ plus normalized threshold opportunistic relay selection (N+NT-ORS) [24] have also been applied. However, these schemes require at least $N$ orthogonal channels to retransmit, resulting in a large loss of spectral efficiency.

We propose a simple but effective scheme coined opportunistic space-time coding (OSTC) to combat the outdated CSI, while avoiding an unnecessary loss of spectral efficiency. A predefined number (i.e., $N$ ) of relays, rather than a single relay in the conventional ORS, are opportunistically selected from $K$ cooperating relays according to instantaneous CSIs of the relay-destination channels. At these selected relays, $N$-dimensional orthogonal space-time block coding (OSTBC) [25] is employed to encode the regenerated signals. Then, $N$ branches coded signals are simultaneously transmitted from the relays to the destination, followed by a simple maximumlikelihood decoding based only on linear processing at the receiver. In contrast to DSTC where all relays participate in the signal's retransmission without any need of selection, only a fraction of relays are opportunistically activated in the proposed scheme. Therefore, opportunistic space-time coding can be regarded as a combination of opportunistic relay selection and distributed space-time coding.

The main contributions of this paper are:

1) A simple and robust opportunistic relaying scheme is proposed for decode-and-forward cooperative systems. It can achieve a remarkable performance gain over the existing schemes in the presence of outdated CSI. From the perspective of multiplexing-diversity trade-off, the proposed scheme is the best solution until now.

2) Closed-form expressions of outage probability and ergodic capacity for OSTC, ORS and GSC are comparatively derived, together with an asymptotic analysis that clarifies the achievable diversity.

3) Monte-Carlo simulations are set up, and numerical results corroborate theoretical analyses.

The remainder of this paper is organized as follows. Section II introduces the system model of a DF cooperative system. Section III presents the proposed scheme. In Section IV, its outage probability and diversity order are theoretically derived, followed by an analysis of ergodic capacity in Section V. Numerical results are given in Section VI. Finally, Section VII concludes this paper.

\section{SySTEM MODEL}

\section{A. Decode-and-forward Cooperative System}

We consider a dual-hop cooperative network [26] where a single source $s$ communicates with a single destination $d$ with the help of $K$ decode-and-forward relays. Suppose that a direct link between the source and destination does not exist owing to a line-of-sight blockage. Because of severe signal attenuations in radio channels, a strong self-interference will be generated if a relay simultaneously transmits and receives signals at the same frequency. It is cost-inefficient to implement a fullduplex transmission upon mobile terminals or wireless nodes with the current radio technology. Consequently, the relays need to operate in a half-duplex mode. It is generally assumed that the relays are equipped with a single antenna due to the limitations of cost, power supply and hardware size on mobile terminals and wireless nodes. Moreover, the spatiallydistributed relays with independent oscillators give rise to multiple timing offset and multiple carrier frequency offset. However, this multi-relay synchronization problem has been well-addressed and reported in the literature, such as [27], [28]. For simplicity, as most of papers in this field, we assume perfect synchronization throughout this paper.

Without loss of generality, the received signal in an arbitrary link $A \rightarrow B$ can be modeled as

$$
y_{B}=h_{A, B} x_{A}+z,
$$

where $x_{A}$ is a transmitted signal with power $E\left[\left|x_{A}\right|^{2}\right]=P_{A}$, and $E[\cdot]$ stands for the statistical mean. Assuming a Rayleigh channel with the block fading, its gain $h_{A, B}$ is zero-mean circularly-symmetric complex Gaussian random variable with variance of $\Omega_{A, B}$, i.e., $h_{A, B} \sim \mathcal{C N}\left(0, \Omega_{A, B}\right)$. Besides, additive white Gaussian noise (AWGN) with zero-mean and variance of $\sigma^{2}$, i.e., $z \sim \mathcal{C N}\left(0, \sigma^{2}\right)$, is applied at the receiver. Node $A$ could be the source $A=s$ or the $k^{\text {th }}$ relay $A=k, k \in\{1, \ldots, K\}$, while $B$ denotes the $k^{\text {th }}$ relay $B=k$ or the destination $B=d$. 
The instantaneous signal-to-noise ratio (SNR) is expressed as $\gamma_{A, B}=\left|h_{A, B}\right|^{2} P_{A} / \sigma^{2}$ and the average $\operatorname{SNR} \bar{\gamma}_{A, B}=\Omega_{A, B} P_{A} / \sigma^{2}$.

In practice, mobile terminals in a cellular network have an upper limit on the transmit power, which is denoted by $P_{u}$ here. To simplify analysis, all links are assumed to be independent and identically distributed (i.i.d.) Rayleigh channels with a normalized gain of $\Omega_{A, B}=1$, i.e., $h \sim \mathcal{C N}(0,1)$. Therefore, the average received SNR for each link is equal to $P_{u} / \sigma^{2}$. In a cooperative system, the total power grows with the increased number of activated relays, imposing an unfairness on different schemes. For instance, a total power of $(N+1) P_{u}$ is consumed by using $N$ selected relays in comparison with $2 P_{u}$ in the case of a single-relay scheme. We call it $(i)$ the practical power mode where either the source or the relays transmit their signals with the fixed power $P_{u}$. In addition, we need to define (ii) the theoretical power mode [29] to facilitate a fair performance comparison. Given an end-to-end power constraint of $P$, we have $P_{s}+\sum_{k=1}^{K} P_{k}=P$, where $P_{s}$ and $P_{k}$ denote the source's and the $k^{\text {th }}$ relay's power, respectively. Thus, a unified average SNR for all source-relay channels can be given by $\bar{\gamma}_{s}=P_{s} / \sigma^{2}$. If the power of activated relays are identical while $P_{k}=0$ for unselected relays, a unified average SNR for the relay-destination channels can be written as

$$
\bar{\gamma}_{N}=\frac{P-P_{s}}{N \sigma^{2}},
$$

or

$$
\bar{\gamma}_{L}=\frac{P-P_{s}}{L \sigma^{2}},
$$

when the number of activated relays are $N$ and $L$, respectively. Similarly, the average SNRs in the practical power mode are expressed as

$$
\bar{\gamma}_{s}=\bar{\gamma}_{N}=\bar{\gamma}_{L}=\frac{P_{u}}{\sigma^{2}} .
$$

\section{B. The Outdated CSI}

From a practical point of view, CSI at the instant of selecting a relay may substantially differ from CSI at the instant of using the selected relay to retransmit. Taking advantage of an outdated version of the CSI $\hat{h}$ rather than the actual CSI $h$ may lead to wrong relay selection, which deteriorates the system performance. To quantify the inaccuracy of outdated CSI, the envelope of the correlation coefficient between $h$ and $\hat{h}$ is defined as

$$
\rho=\frac{|\operatorname{cov}(h, \hat{h})|}{\mu_{h} \mu_{\hat{h}}},
$$

where $\operatorname{cov}(\cdot)$ stands for the covariance of two random variables, and $\mu$ is the standard deviation. Derived from Eq. (3) of [30], we have

$$
h=\rho \hat{h}+\varepsilon \sqrt{1-\rho^{2}},
$$

where $\varepsilon$ is a normalized Gaussian random variable, i.e., $\varepsilon \sim \mathcal{C N}(0,1)$. The actual CSI $h$ and its outdated version $\hat{h}$ follow joint Gaussian distribution. Thus, $h$ conditioned on $\hat{h}$ is also Gaussian distributed:

$$
h \mid \hat{h} \sim \mathcal{C N}\left(\rho \hat{h}, 1-\rho^{2}\right) .
$$

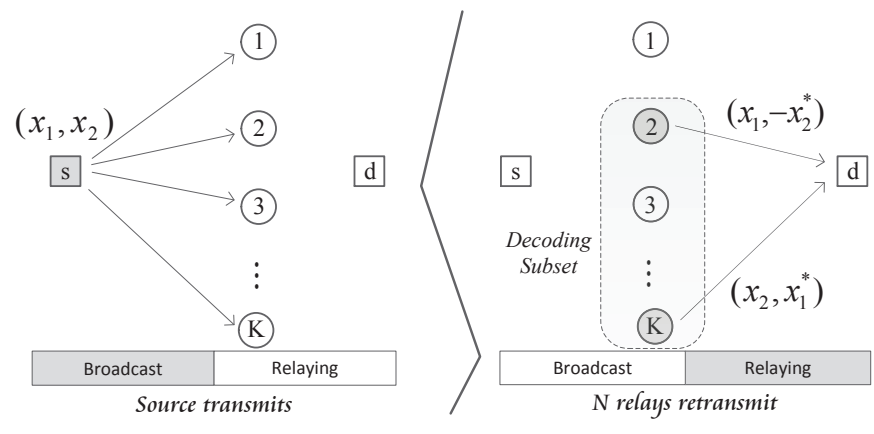

Fig. 1. Schematic diagram of the proposed scheme. For illustration purposes, we use $N=2$ as an example. In the broadcast phase, the source transmits a pair of symbols $\left(x_{1}, x_{2}\right)$ in two consecutive symbol periods. In the relaying phase, two relays with strongest CSIs in relay-destination channels are selected from the decoding subset, and the Alamouti scheme is applied to encode the regenerated symbols at this pair of relays. Then, a relay transmits $\left(x_{1},-x_{2}^{*}\right)$ while another relay sends $\left(x_{2}, x_{1}^{*}\right)$ simultaneously at the same frequency.

Due to the assumption of a normalized channel gain $\Omega_{A, B}=1$, the average SNR is simplified to $\bar{\gamma}_{A, B}=P_{A} / \sigma^{2}$. Thus, the instantaneous SNR can be rewritten as $\gamma_{A, B}=\left|h_{A, B}\right|^{2} \bar{\gamma}_{A, B}$. Conditioned on its outdated version $\hat{\gamma}_{A, B}=\left|\hat{h}_{A, B}\right|^{2} \bar{\gamma}_{A, B}, \gamma_{A, B}$ follows a noncentral Chi-square distribution with two degrees of freedom. As given in Eq. (12) of [31], its probability density function (PDF) is

$$
\begin{aligned}
& f_{\gamma_{A, B} \mid \hat{\gamma}_{A, B}}(\gamma \mid \hat{\gamma})= \\
& \frac{1}{\bar{\gamma}_{A, B}\left(1-\rho^{2}\right)} e^{-\frac{\gamma+\rho^{2} \hat{\gamma}}{\bar{\gamma}_{A, B}\left(1-\rho^{2}\right)}} I_{0}\left(\frac{2 \sqrt{\rho^{2} \gamma \hat{\gamma}}}{\bar{\gamma}_{A, B}\left(1-\rho^{2}\right)}\right),
\end{aligned}
$$

where $I_{0}(\cdot)$ is the zero $^{\text {th }}$ order modified Bessel function of the first kind.

\section{Opportunistic Space-Time Coding}

The fundamental principle of opportunistic space-time coding is that a predefined number of relays are opportunistically selected and then the selected relays simultaneously retransmit at the same frequency by means of space-time block coding.

\section{A. The Rationale of OSTC}

Because of the severe attenuation of radio signals, a singleantenna relay has to operate in a half-duplex mode to avoid harmful self-interference between the transmitter and receiver. Without loss of generality, the signal transmission can be realized within two time slots: the broadcast and relaying phases. In the broadcast phase, the source transmits and those relays who can correctly decode the original signal constitute a decoding subset $\mathcal{D S}$ :

$$
\begin{aligned}
\mathcal{D} \mathcal{S} & \triangleq\left\{k: \frac{1}{2} \log _{2}\left(1+\gamma_{s, k}\right) \geqslant R\right\} \\
& =\left\{k: \gamma_{s, k} \geqslant 2^{2 R}-1\right\} \\
& =\left\{k: \gamma_{s, k} \geqslant \gamma_{t h}\right\},
\end{aligned}
$$

where $\hat{\gamma}_{s, k}$ is the SNR of source-relay channel, $R$ is an endto-end target rate for the dual-hop relaying and $\gamma_{t h}=2^{2 R}-1$ denotes the threshold SNR. It is noted that the required rate for each hop doubles to $2 R$ owing to the half-duplex mode. 
In the conventional ORS [12], the relay having the strongest SNR (interchangeable with CSI if the given transmit power for each relay is equal) in the relay-destination channels is selected from $\mathcal{D S}$ to serve as the best relay $\tilde{k}$, i.e.,

$$
\tilde{k}=\arg \max _{k \in \mathcal{D} \mathcal{S}} \hat{\gamma}_{k, d},
$$

where $\hat{\gamma}_{k, d}$ is the SNR of relay-destination channel at the instant of selecting relay, which may be outdated in comparison with the actual SNR $\gamma_{k, d}$ at the instant of using the selected relay to retransmit. In DSTC [10], no relay selection process is performed, where all relays belonging to $\mathcal{D S}$ are used to retransmit. In this case, the number of participating relays is unknown and randomly varying since $\mathcal{D S}$ dynamically changes with the fluctuation of radio channels. Instead of selecting a single relay in ORS or all-participating in DSTC, the proposed scheme chooses a fixed number of relays, i.e.,

$$
\begin{aligned}
& \tilde{k}_{1}=\arg \max _{k \in \mathcal{D S}} \hat{\gamma}_{k, d}, \\
& \tilde{k}_{n}=\arg \max _{k \in \mathcal{D S}-\left\{\tilde{k}_{1}, \ldots, \tilde{k}_{n-1}\right\}} \hat{\gamma}_{k, d}, \quad 2 \leqslant n \leqslant N .
\end{aligned}
$$

In the relaying phase, an $N$-dimensional orthogonal spacetime block code is applied to encode the regenerated signals at the selected relays $\tilde{k}_{n}, 1 \leqslant n \leqslant N$ in a distributed manner. $N$ branches coded signals are simultaneously transmitted by the selected relays at the same frequency, followed by a simple maximum-likelihood decoding based only on linear processing at the receiver. If the number of relays in a decoding subset is denoted by $L$, we have $0 \leqslant L \leqslant K$. It is possible that $L$ is less than the predefined number of $N$, i.e., $L<N$. In this case, all of $L$ relays participate in the signal retransmission directly, without any relay selection, to lower the complexity and increase the achievable diversity.

For illustration purposes, we select $N=2$ and use the Alamouti scheme [32] to clarify the proposed scheme. In the broadcast phase, as illustrated in Fig.1, the source sends a pair of symbols $\left(x_{1}, x_{2}\right)$ to all relays within two consecutive symbol periods. Those relays who correctly decode the original signal constitute a decoding subset. In accordance to instantaneous SNRs of the relay-destination channels, a pair of relays $\left(\tilde{k}_{1}, \tilde{k}_{2}\right)$ are opportunistically selected. In the relaying phase, the regenerated symbols are space-time encoded as:

$$
\left(x_{1}, x_{2}\right) \Longrightarrow\left[\begin{array}{rr}
x_{1} & -x_{2}^{*} \\
x_{2} & x_{1}^{*}
\end{array}\right],
$$

where the superscript $*$ denotes the complex conjugate. Then, a relay transmits the first branch of coded symbols $\left(x_{1},-x_{2}^{*}\right)$, while another relay sends $\left(x_{2}, x_{1}^{*}\right)$ simultaneously at the same frequency, analogous to the Alamouti scheme applying for two co-located antennas. At the destination, the received signals $\left(y_{1}, y_{2}\right)$ in two consecutive symbol periods can be written as

$$
\begin{aligned}
& y_{1}=h_{\tilde{k}_{1}, d} x_{1}+h_{\tilde{k}_{2}, d} x_{2}+z_{1} \\
& y_{2}=h_{\tilde{k}_{2}, d} x_{1}^{*}-h_{\tilde{k}_{1}, d} x_{2}^{*}+z_{2} .
\end{aligned}
$$

Through a simple maximum-likelihood decoding based on linear processing [32], the transmitted symbols can be recovered:

$$
\begin{aligned}
x_{1}^{\prime} & =\left(\left|h_{\tilde{k}_{1}, d}\right|^{2}+\left|h_{\tilde{k}_{2}, d}\right|^{2}\right) x_{1}+\tilde{z}_{1} \\
x_{2}^{\prime} & =\left(\left|h_{\tilde{k}_{1}, d}\right|^{2}+\left|h_{\tilde{k}_{2}, d}\right|^{2}\right) x_{2}+\tilde{z}_{2} .
\end{aligned}
$$

The gains $h_{\tilde{k}_{1}, d}$ and $h_{\tilde{k}_{2}, d}$ can be acquired by the channel estimation at the receiver. Note that the impact of channel estimation errors on the signal detection is not considered in this article since it is negligible compared with the effect of outdated CSI on the relay selection.

We return to the general cases where $N$-dimensional OSTBC is applied for $N$ selected relays. According to [25], [32], OSTBC can reap a full diversity for an arbitrary dimension of $N$, but only the Alamouti scheme achieves both full-rate and full-diversity with complex signal constellations. For $N=3$ and 4 , there exists a capacity loss since a maximal rate of $3 / 4$ is realized. To get a higher diversity of $N>4$, only a rate of $1 / 2$ can be kept. Hence, we need a factor $\beta$ to model this capacity loss, which is defined as

$$
\beta_{n}= \begin{cases}1, & n=1,2 \\ 0.75, & n=3,4 \\ 0.5, & n>4\end{cases}
$$

Based on this factor, a unified form of the capacity for the relay-destination channel can be given by

$$
C_{0}^{(N)}=\beta_{N} \cdot \log _{2}\left(1+\sum_{n=1}^{N} \frac{\left|h_{\tilde{k}_{n}, d}\right|^{2} P_{\tilde{k}_{n}}}{\sigma^{2}}\right),
$$

where $\log _{2}(\cdot)$ is the binary logarithm, $h_{\tilde{k}_{n}, d}$ is the channel gain from the relay $\tilde{k}_{n}$ to the destination, and $P_{\tilde{k}_{n}}$ stands for its transmit power.

Last but not least, the capacity loss of OSTBC also affects the threshold SNR defined in (4), which needs to be accordingly modified to

$$
\gamma_{t h}^{n}=2^{2 R / \beta_{n}}-1 .
$$

\section{B. An Example of Implementation}

The implementation of opportunistic relaying schemes can be divided into two categories: the centralized [11] or distributed [33] methods. The former relies on a central controller, e.g., the destination, which collects the global CSIs, carries out the relay selection, and informs the selected relays to retransmit. The information exchange between the central controller and the relays not only requires a large amount of signaling, but also brings the feedback delay that exacerbates the outdated CSI problem. To justify the feasibility of the proposed scheme, we give an example of distributed implementation, as follows:

1) The source broadcasts a short packet called Ready-ToSend (RTS), followed by Clear-to-Send (CTS) from the destination [11]. Based on RTS and CTS, each relay can estimate the corresponding channel gains.

2) Once receiving the CTS, a timer with duration inversely proportional to the channel gain is started at each relay.

3) The relay with the largest channel gain expires first, who sends a short packet to announce its presence. Then, the second relay reports its presence when its timer expires. This process iterates until $N$ relays are selected.

4) Once receiving the $N^{t h}$ relay's packet of its presence, the selected relays retransmit simultaneously.

It is possible that the number of relays in a decoding subset is less than $N$ or the duration of timer is too long due to a 
very small channel gain. For these cases, we can further set a threshold (maximal) duration. If this duration expires, the relay selection process is interrupted regardless of the number of presented relays.

\section{OUtage Probability and Diversity ANALYSis}

In this section, the closed-form expressions of outage probabilities for the proposed scheme and the conventional ORS are derived by means of an moment generating function (MGF)-based performance analysis approach, together with an asymptotic analysis that clarifies their achievable diversity.

\section{A. Outage Probability}

An outage points to that the instantaneous channel capacity falls below a target rate $R$, i.e., $C<R$, where a reliable communication is impossible whatever coding used. To measure this outage, the outage probability [1], an important performance metric over fading channels, is defined as

$$
P_{\text {out }}(R)=\operatorname{Pr}\left\{\log _{2}(1+\gamma)<R\right\},
$$

where $\operatorname{Pr}$ is the notation of mathematical probability.

1) OSTC with the Outdated CSI: In the proposed scheme, if all relays fail to decode the original signal in the sourcerelay channels, i.e., $L=0$, or the overall received SNR at the destination is less than the threshold SNR, an outage occurs.

Because of the channel fading, the number of relays that can successfully decode the original signal randomly varies in the range of 0 to $K$. Denoting all decoding subsets that contain $L$ relays by a set $\mathcal{D} \mathcal{S}_{L}$, we have $\mathcal{D} \mathcal{S}_{L}=\left\{\mathcal{D} \mathcal{S}_{L}^{p}: p=1, \ldots,\left|\mathcal{D} \mathcal{S}_{L}\right|\right\}$, where $\mathcal{D} \mathcal{S}_{L}^{p}$ is the $p^{\text {th }}$ element of $\mathcal{D} \mathcal{S}_{L}$ and $|\cdot|$ represents the cardinality of a set. Conditioned on $\mathcal{D} \mathcal{S}_{L}^{p}$, OSTC's outage probability can be computed as

$$
P_{\text {ostc }}\left(\gamma_{t h}\right)=\sum_{L=0}^{K} \sum_{p=1}^{\left|\mathcal{D} \mathcal{S}_{L}\right|} \operatorname{Pr}\left(\text { outage } \mid \mathcal{D} \mathcal{S}_{L}^{p}\right) \operatorname{Pr}\left(\mathcal{D} \mathcal{S}_{L}^{p}\right),
$$

where $\operatorname{Pr}\left(\mathcal{D S} \mathcal{S}_{L}^{p}\right)$ denotes the occurrence probability of $\mathcal{D} \mathcal{S}_{L}^{p}$, and $\operatorname{Pr}\left(\right.$ outage $\left.\mid \mathcal{D} \mathcal{S}_{L}^{p}\right)$ is the outage probability conditioned on $\mathcal{D} \mathcal{S}_{L}^{p}$. Recalling the assumption of i.i.d. Rayleigh-faded channels, the values of $\operatorname{Pr}\left(\mathcal{D} \mathcal{S}_{L}^{p}\right)$ are identical for any $p \in\left\{1, \ldots,\left|\mathcal{D} \mathcal{S}_{L}\right|\right\}$, and as well $\operatorname{Pr}\left(\right.$ outage $\left.\mid \mathcal{D} \mathcal{S}_{L}^{p}\right)$. Hence, (12) can be simplified to

$$
P_{\text {ostc }}\left(\gamma_{t h}\right)=\sum_{L=0}^{K} \operatorname{Pr}(\text { outage } \mid L) \operatorname{Pr}(|\mathcal{D S}|=L),
$$

where $\operatorname{Pr}(|\mathcal{D S}|=L)$ denotes the occurrence probability that the current decoding subset has $L$ relays, and $\operatorname{Pr}($ outage $\mid L)$ is the outage probability conditioned on $L$.

The instantaneous SNR of each source-relay Rayleigh channel is exponentially distributed, i.e., $\gamma_{s, k} \sim \operatorname{EXP}\left(\frac{1}{\bar{\gamma}_{s}}\right)$, where $\bar{\gamma}_{s}$ is a unified average SNR as mentioned in (1) and (2). Its cumulative distribution function (CDF) can be given by

$$
F_{\gamma_{s, k}}(x)=1-e^{-\frac{x}{\gamma_{s}}}, \quad x>0 .
$$

From (4) and (14), we get the probability that a relay correctly decodes the original signal and falls into $\mathcal{D S}$, which is equal to $1-F_{\gamma_{s, k}}\left(\gamma_{t h}\right)$. Following the definition of Binomial distribution, we can get the occurrence probability of successfully decoding $L$ out of $K$ relays, i.e.,

$$
\operatorname{Pr}(|\mathcal{D S}|=L)=\left(\begin{array}{c}
K \\
L
\end{array}\right)\left(e^{-\frac{\gamma_{t h}}{\gamma_{s}}}\right)^{L}\left(1-e^{-\frac{\gamma_{t h}}{\gamma_{s}}}\right)^{K-L} .
$$

On the other hand, the outage probability $\operatorname{Pr}$ (outage $\mid L)$ can be derived conditioned on different values of $L$, as follows:

a) $L=0$ : In this case, all relays fail to decode the source's signal, which is an outage, i.e.,

$$
\operatorname{Pr}(\text { outage } \mid 0)=1 .
$$

b) $1 \leqslant L \leqslant N$ : When the number of available relays in a decoding subset $\mathcal{D S}$ is not larger than the predefined number $N$, all of $L$ relays, i.e., $\tilde{k}_{1}, \tilde{k}_{2}, \ldots, \tilde{k}_{L}$, directly participate in the signal relaying without the involvement of relay selection.

The total received SNR at the destination can be denoted by $\gamma_{t o t}^{L}=\sum_{n=1}^{L} \gamma_{\tilde{k}_{n}, d}$, where $\gamma_{\tilde{k}_{n}, d}=\left|h_{\tilde{k}_{n}, d}\right|^{2} P_{\tilde{k}_{n}} / \sigma^{2}$ stands for the channel SNR from the relay $\tilde{k}_{n}$ to the destination. Recalling (1) and (2), the SNR $\gamma_{\tilde{k}_{n}, d}$ is exponentially distributed with an average SNR of $\bar{\gamma}_{\bar{k}_{n}, d}=\bar{\gamma}_{L}$. According to [34], this total SNR $\gamma_{\text {tot }}^{L}$ follows the Erlang distribution, whose CDF is

$$
F_{\gamma}(x)=1-\sum_{n=1}^{L} \frac{1}{(n-1) !} e^{-\frac{x}{\bar{\gamma}_{L}}}\left(\frac{x}{\bar{\gamma}_{L}}\right)^{n-1},
$$

where $(n-1)$ ! is the notation of factorial that is the product of all the integers from 1 to $n-1$. In this case, the threshold SNR $\gamma_{t h}^{L}$ can be obtained by (11) for $n=L$, i.e., $\gamma_{t h}^{L}=2^{2 R / \beta_{L}}-1$. Substituting $x=\gamma_{t h}^{L}$ into (17), we have

$$
\operatorname{Pr}(\text { outage } \mid L)=1-\sum_{n=1}^{L} \frac{1}{(n-1) !} e^{-\frac{\gamma_{t h}^{L}}{\bar{\gamma}_{L}}}\left(\frac{\gamma_{t h}^{L}}{\bar{\gamma}_{L}}\right)^{n-1} .
$$

It is noted that the outage probabilities (16) and (18) are independent of the outdated CSI, since the relay selection is not utilized in the case of $L \leqslant N$.

c) $L>N$ : If the number of available relays in $\mathcal{D S}$ is more than the predefined number $N$, the relay selection needs to be carried out. A total of $N$ relays, i.e., $\tilde{k}_{1}, \tilde{k}_{2}, \ldots, \tilde{k}_{N}$, which have the largest SNRs in the relay-destination channels, are chosen to retransmit. Using a simple maximum-likelihood decoding, the total SNR at the destination is $\gamma_{t o t}^{N}=\sum_{n=1}^{N} \gamma_{\tilde{k}_{n, d},}$.

The typical approach to derive an outage probability is to first find the received SNR's PDF $f(\gamma)$ and then integrate over this PDF as:

$$
P_{\text {out }}\left(\gamma_{t h}\right)=\int_{0}^{\gamma_{t h}} \log _{2}(1+\gamma) f(\gamma) d \gamma .
$$

However, $f(\gamma)$ is not available in a simple and closed form, so the PDF-based performance analysis is usually mathematically intractable. To be specific, $f(\gamma)$ in OSTC is a nonlinear function of not only the SNRs $\gamma_{1, d}, \gamma_{2, d}, \ldots, \gamma_{K, d}$ but also their outdated versions $\hat{\gamma}_{1, d}, \hat{\gamma}_{2, d}, \ldots, \hat{\gamma}_{K, d}$. Additionally, $N$ relays need to choose according to the outdated SNRs, while the total received SNR is a combination of the actual SNRs, which is an NchooseK problem. Therefore, the derivation of a closedform expression for an outage probability is tedious, if not infeasible. In contrast, an moment generating function of the 


$$
\begin{aligned}
P_{\text {ostc }}\left(\gamma_{t h}\right) & =\left(1-e^{-\frac{\gamma_{t h}}{\gamma_{s}}}\right)^{K} \\
& +\sum_{L=1}^{N}\left(\begin{array}{c}
K \\
L
\end{array}\right)\left(e^{-\frac{\gamma_{t h}}{\bar{\gamma}_{s}}}\right)^{L}\left(1-e^{-\frac{\gamma_{t h}}{\bar{\gamma}_{s}}}\right)^{K-L}\left(1-\sum_{n=1}^{L} \frac{1}{(n-1) !} e^{-\frac{\gamma_{t h}^{L}}{\bar{\gamma}_{L}}}\left(\frac{\gamma_{t h}^{L}}{\bar{\gamma}_{L}}\right)^{n-1}\right) \\
& +\sum_{L=N+1}^{K}\left(\begin{array}{c}
K \\
L
\end{array}\right)\left(e^{-\frac{\gamma_{t h}}{\bar{\gamma}_{s}}}\right)^{L}\left(1-e^{-\frac{\gamma_{t h}}{\bar{\gamma}_{s}}}\right)^{K-L}\left(\frac{2^{-Q} e^{A / 2}}{\gamma_{t h}^{N}} \sum_{q=0}^{Q}\left(\begin{array}{c}
Q \\
q
\end{array}\right) \sum_{p=0}^{P+q} \frac{(-1)^{p}}{\xi_{p}} \mathcal{R}\left\{\frac{\mathcal{M}_{\gamma}\left(\frac{A+2 \pi j p}{2 \gamma_{t h}^{N}}\right)}{\frac{A+2 \pi j p}{2 \gamma_{t h}^{N}}}\right\}\right)
\end{aligned}
$$

$$
\begin{aligned}
C_{o s t c} & =\sum_{L=1}^{N} \frac{\beta_{L}}{\ln (2)\left(1+\beta_{L}\right)}\left(\begin{array}{c}
K \\
L
\end{array}\right)\left(e^{-\frac{\gamma_{t h}}{\bar{\gamma}_{s}}}\right)^{L}\left(1-e^{-\frac{\gamma_{t h}}{\bar{\gamma}_{s}}}\right)^{K-L} \sum_{t=1}^{T}-L \bar{\gamma}_{L} w_{t} \Lambda\left(s_{t}\right)\left(\frac{1}{1+s_{t} \bar{\gamma}_{L}}\right)^{L+1} \\
& +\sum_{L=N+1}^{K} \frac{\beta_{N} \bar{\gamma}_{N} L(L-N) !}{\ln (2)\left(1+\beta_{N}\right)}\left(\begin{array}{c}
L-1 \\
N-1
\end{array}\right)\left(\begin{array}{c}
K \\
L
\end{array}\right)\left(e^{-\frac{\gamma_{t h}}{\bar{\gamma}_{s}}}\right)^{L}\left(1-e^{-\frac{\gamma_{t h}}{\bar{\gamma}_{s}}}\right)^{K-L} \\
& \times \sum_{t=1}^{T} \sum_{m=0}^{L-N} \frac{(-1)^{m+1} w_{t} \Lambda\left(s_{t}\right)}{m !(L-N-m) !}\left(\frac{1}{1+s_{t} \bar{\gamma}_{N}}\right)^{N}\left(\frac{N-1}{\eta\left(s_{t}\right)}+\frac{\left(N+m\left(1-\rho^{2}\right)\right)\left(1+s_{t} \bar{\gamma}_{N}\right)}{\eta^{2}\left(s_{t}\right)}\right)
\end{aligned}
$$

total SNR can be expressed more simply. As given in Eq. (11) of [35], an outage probability can be mapped directly from its MGF as

$$
\begin{aligned}
P_{\text {out }}\left(\gamma_{\text {th }}\right) & =\frac{2^{-Q} e^{A / 2}}{\gamma_{t h}} \sum_{q=0}^{Q}\left(\begin{array}{c}
Q \\
q
\end{array}\right) \sum_{p=0}^{P+q} \frac{(-1)^{p}}{\xi_{p}} \\
& \times \mathcal{R}\left\{\frac{\mathcal{M}_{\gamma}\left(\frac{A+2 \pi j p}{2 \gamma_{t h}}\right)}{\frac{A+2 \pi j p}{2 \gamma_{t h}}}\right\}+E(A, P, Q),
\end{aligned}
$$

where $j^{2}=-1, \mathcal{R}\{\cdot\}$ denotes the real part, $\mathcal{M}_{\gamma}(\cdot)$ is the MGF of the total SNR, $A, P$ and $Q$ are the numerical parameters, which satisfy the condition that an error term $E(A, P, Q)$ is negligible compared with the outage probability. As recommended by [35], using $A=10 \ln 10, P=21$ and $Q=15$ can result in $E(A, P, Q)<10^{-10}$. Besides, the coefficient $\xi_{p}$ is

$$
\xi_{p}=\left\{\begin{array}{l}
2, p=0 \\
1, p=1,2, \ldots
\end{array}\right.
$$

From (22), we know that $\operatorname{Pr}($ outage $\mid L)$ could be obtained once the MGF of $\gamma_{\text {tot }}^{N}$ is available. Making full use of a probability space partition approach proposed in [8] and [24], this MGF can be obtained:

Lemma 1: In the case of $L>N$, the moment generating function for the total SNR $\gamma_{t o t}^{N}$ can be expressed as

$$
\begin{aligned}
\mathcal{M}_{\gamma}(s) & =L(L-N) !\left(\begin{array}{c}
L-1 \\
N-1
\end{array}\right)\left(\frac{1}{1+s \bar{\gamma}_{N}}\right)^{N-1} \\
& \times \sum_{m=0}^{L-N} \frac{(-1)^{m}}{m !(L-N-m) !} \cdot \frac{1}{\eta(s)}
\end{aligned}
$$

where the average SNR $\bar{\gamma}_{N}$ is defined in (1) and (2), and

$$
\eta(s)=N\left(1+s \bar{\gamma}_{N}\right)+m\left[1+s \bar{\gamma}_{N}\left(1-\rho^{2}\right)\right] .
$$

Proof: See Appendix A.
Substituting (23) into (22), yields

$$
\begin{aligned}
\operatorname{Pr}(\text { outage } \mid L) & =\frac{2^{-Q} e^{A / 2}}{\gamma_{t h}^{N}} \sum_{q=0}^{Q}\left(\begin{array}{c}
Q \\
q
\end{array}\right) \\
& \times \sum_{p=0}^{P+q} \frac{(-1)^{p}}{\xi_{p}} \mathcal{R}\left\{\frac{\mathcal{M}_{\gamma}\left(\frac{A+2 \pi j p}{2 \gamma_{t h}^{N}}\right)}{\frac{A+2 \pi j p}{2 \gamma_{t h}^{N}}}\right\},
\end{aligned}
$$

where the threshold SNR is $\gamma_{t h}^{N}=2^{2 R / \beta_{N}}-1$ in terms of (11).

Looking back to (13), $\operatorname{Pr}(|\mathcal{D} \mathcal{S}|=L)$ and $\operatorname{Pr}$ (outage $\mid L)$ are figured out, so the overall outage probability $P_{o s t c}\left(\gamma_{t h}\right)$ can be derived:

Theorem 1: In the presence of outdated CSI, the outage probability for the proposed OSTC scheme over i.i.d. Rayleigh channels can be given in a closed form by (20).

Proof: By substituting (15), (16), (18) and (25) into (13), yields (20).

2) ORS with the Outdated CSI: Interestingly, the proposed scheme can be transformed to the ORS if the number of selected relays is $N=1$. Accordingly, ORS's outage probability $P_{\text {ors }}\left(\gamma_{t h}\right)$ is obtained by substituting $N=1$ into (20), i.e.,

$$
P_{\text {ors }}\left(\gamma_{t h}\right)=\left.P_{\text {ostc }}\left(\gamma_{t h}\right)\right|_{N=1} \text {. }
$$

In [13], the outage probability of ORS in the presence of outdated CSI has already been derived through the PDF-based performance analysis. It is easy to verify that the outage probability given by the MGF-based expression of (26) tightly matches the PDF-based expression of Eq. (2) in [13]. This can partly corroborate our theoretical analyses in this section.

\section{B. Asymptotic Diversity Analysis}

From (20), it is still difficult to make an insightful comparison among different schemes. It is worth providing an asymptotic analysis in high SNR regime to clarify their achievable 
diversity, as the definition of $d=-\lim _{\bar{\gamma} \rightarrow \infty} \log \left(P_{\text {out }}\right) / \log (\bar{\gamma})$ in [13]. Both ORS [13] and GSC [24] can achieve a full diversity of $K$ with the prefect CSI. However, their diversity are decreased to 1 and $N$, respectively, in the presence of outdated CSI. For the proposed scheme, we have

Theorem 2: The achievable diversity order of the proposed scheme is

$$
d= \begin{cases}N, & \rho<1 \\ K, & \rho=1\end{cases}
$$

Proof: See Appendix B.

\section{CAPACITy ANALYSis}

The ergodic capacity is defined as [1]:

$$
C=\int_{0}^{\infty} \log _{2}(1+\gamma) f(\gamma) d \gamma
$$

that is another key performance metric to indicate the highest rate at which a signal can be transmitted over a radio channel with a negligible error probability. As mentioned in the previous section, $f(\gamma)$ is difficult to be obtained in a simple and closed form. To avoid intractability of PDF-based performance analysis, we take advantage of an MGF-based approach [36], as follows:

Lemma 2: The ergodic capacity can be calculated through the MGF of the received SNR, given by:

$$
C=\frac{1}{\ln (2)} \sum_{t=1}^{T} w_{t} \Lambda\left(s_{t}\right)\left[\left.\frac{\partial}{\partial s} M_{\gamma}(s)\right|_{s \rightarrow s_{t}}\right],
$$

where $\ln$ denotes the natural logarithm, $\frac{\partial}{\partial s}$ expresses the partial derivative with respect to $s, T$ is a truncation index (setting $T=200$ is accurate enough for SNRs lower than $30 \mathrm{~dB}), \Lambda(x)$ stands for a special function called Meijer's $\mathrm{G}^{1}$,

$$
\Lambda(s)=-G_{2,1}^{0,2}\left[\begin{array}{l|l}
1,1 & \frac{1}{s} \\
0 & s
\end{array}\right]
$$

and the coefficients $s_{t}$ and $w_{t}$ are defined as

$$
\begin{aligned}
& s_{t}=\tan \left[\frac{\pi}{4} \cos \left(\frac{2 t-1}{2 T} \pi\right)+\frac{\pi}{4}\right], \\
& w_{t}=\frac{\pi^{2} \sin \left(\frac{2 t-1}{2 T} \pi\right)}{4 T \cos ^{2}\left[\frac{\pi}{4} \cos \left(\frac{2 t-1}{2 T} \pi\right)+\frac{\pi}{4}\right]} .
\end{aligned}
$$

Proof: The detailed derivation can be found in [36].

Subsequently, we make full use of this MGF-based approach to figure out the proposed scheme's capacity. Meanwhile, the closed-form capacity expressions for ORS and GSC are also derived, which are still not available in the literature until now.

\footnotetext{
${ }^{1}$ The capacity in [16] contains an exponential integral $\int_{1}^{\infty} t^{-1} e^{-\lambda t} d t$, whose result is still a function of $\lambda$. In contrast, the result of Meijer's $G$ is a numerical value, e.g., $G_{2,1}^{0,2}\left[\begin{array}{l}1,1 \\ 0\end{array} \mid 1\right] \approx 0.2193839$, leading to a closedform expression. The mathematical softwares such as MATHEMATICA ${ }^{\circledR}$ and MATLAB ${ }^{\circledR}$ have already implemented Meijer's $G$ in their in-build function library. That is the motivation of using the MGF-based method here.
}

\section{A. Capacity of OSTC}

Analogous to (13), the end-to-end ergodic capacity of the proposed scheme can be computed by:

$$
C_{\text {ostc }}=\sum_{L=0}^{K} C^{L} \operatorname{Pr}(|\mathcal{D S}|=L),
$$

where $\operatorname{Pr}(|\mathcal{D S}|=L)$ is the occurrence probability given in (15), and $C^{L}$ denotes the end-to-end capacity conditioned on $|\mathcal{D S}|=L$, which can be derived as follows:

a) $L \leqslant N$ : If the number of available relays in $\mathcal{D S}$ is not larger than the predefined number $N$, all of $L$ relays directly participate in the signal retransmission without the need of relay selection. For a Rayleigh-faded channel, its MGF with respect to the received SNR $\gamma_{\tilde{k}_{n}, d}$ is given by

$$
\mathcal{M}_{\gamma_{n}}(s)=\frac{1}{1+s \bar{\gamma}_{\tilde{k}_{n}, d}},
$$

where $\bar{\gamma}_{\tilde{k}_{n}, d}$ is the average SNR from relay $\tilde{k}_{n}$ to the destination. At the receiver, the total SNR can be denoted by $\gamma_{\text {tot }}^{L}=\sum_{n=1}^{L}\left|h_{\tilde{k}_{n}, d}\right|^{2} P_{\tilde{k}_{n}} / \sigma^{2}$. According to [36], the MGF of $\gamma_{\text {tot }}^{L}$ is equal to the multiplication of the MGFs for all links, i.e., $\mathcal{M}_{\gamma}^{L}(s)=\prod_{n=1}^{L} \mathcal{M}_{\gamma_{n}}(s)$. We have

$$
\mathcal{M}_{\gamma}^{L}(s)=\prod_{n=1}^{L} \frac{1}{1+s \bar{\gamma}_{\tilde{k}_{n}, d}} .
$$

As defined in (1) and (2), all relay-destination channels have a unified average SNR $\bar{\gamma}_{L}$. Thus, (30) can be simplified to

$$
\mathcal{M}_{\gamma}^{L}(s)=\left(\frac{1}{1+s \bar{\gamma}_{L}}\right)^{L} .
$$

Different from the end-to-end capacity $C^{L}$, the capacity of the relay-destination channel is denoted by $C_{0}^{L}$. Substituting (31) into (27), $C_{0}^{L}$ rather than $C^{L}$ can be figured out. It is noted that there exists a capacity loss owing to the utilization of orthogonal space-time block coding, as depicted by the factor $\beta$ in (9). Moreover, the dual-hop cooperative system uses an extra time slot for the broadcast phase, which can be modelled by a modified factor of $\beta /(1+\beta)$. The end-to-end capacity for the case of $L \leqslant N$ can be computed as

$$
C^{L}=\frac{\beta_{L}}{1+\beta_{L}} C_{0}^{L} .
$$

b) $L>N$ : When the number of available relays in $\mathcal{D S}$ is more than the predefined number $N$, the total received SNR is $\gamma_{t o t}^{N}=\sum_{n=1}^{N}\left|h_{\tilde{k}_{n}, d}\right|^{2} P_{\tilde{k}_{n}} / \sigma^{2}$, whose MGF is given in (23). The first partial derivative of (23) is

$$
\begin{aligned}
\frac{\partial \mathcal{M}_{\gamma}(s)}{\partial s} & =\bar{\gamma}_{N} L(L-N) !\left(\begin{array}{c}
L-1 \\
N-1
\end{array}\right)\left(\frac{1}{1+s \bar{\gamma}_{N}}\right)^{N} \\
& \times \sum_{m=0}^{L-N} \frac{(-1)^{m+1}}{m !(L-N-m) !} \\
& \cdot\left(\frac{N-1}{\eta(s)}+\frac{\left(N+m\left(1-\rho^{2}\right)\right)\left(1+s \bar{\gamma}_{N}\right)}{\eta^{2}(s)}\right) .
\end{aligned}
$$




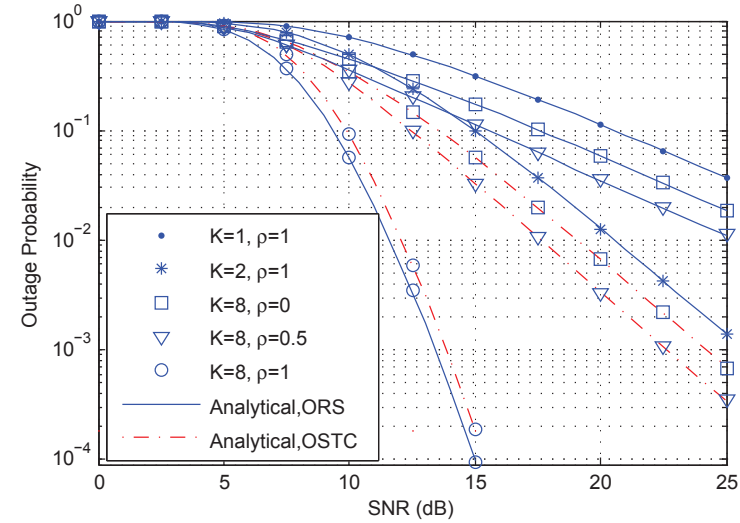

(a)

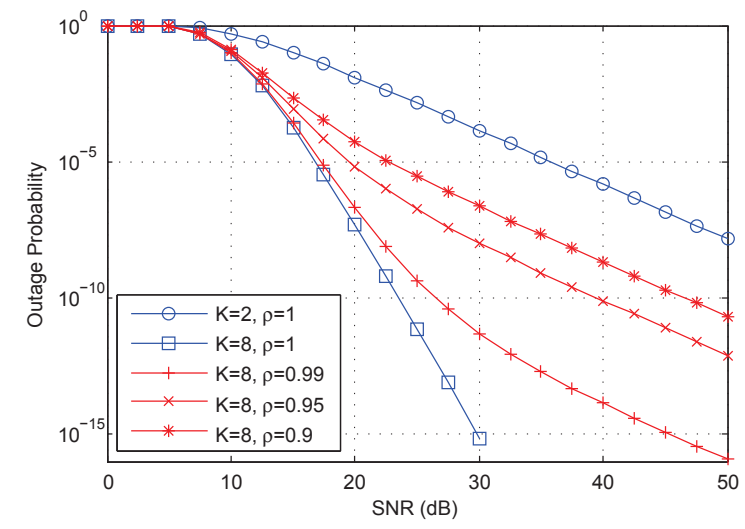

(b)

Fig. 2. (a) Outage probabilities of OSTC and ORS as a function of the average SNR $\bar{\gamma}=P / \sigma^{2}$ in the case of $K=8$ and $N=2$; (b) Outage probabilities of OSTC in the high SNR using $K=8$ and $N=2$. Numerical results are marked by markers while analytical results by curves.

Substituting (33) into (27), the capacity of the relay-destination channel is obtained. Analogous to (32), a modified factor $\beta_{N} /\left(1+\beta_{N}\right)$ is needed, yielding:

$$
\begin{aligned}
C^{L} & =\frac{\beta_{N}}{1+\beta_{N}} \cdot \frac{\bar{\gamma}_{N} L(L-N) !}{\ln (2)}\left(\begin{array}{c}
L-1 \\
N-1
\end{array}\right) \\
& \times \sum_{t=1}^{T} \sum_{m=0}^{L-N} \frac{(-1)^{m+1} w_{t} \Lambda\left(s_{t}\right)}{m !(L-N-m) !}\left(\frac{1}{1+s_{t} \bar{\gamma}_{N}}\right)^{N} \\
& \times\left(\frac{N-1}{\eta\left(s_{t}\right)}+\frac{\left(N+m\left(1-\rho^{2}\right)\right)\left(1+s_{t} \bar{\gamma}_{N}\right)}{\eta^{2}\left(s_{t}\right)}\right) .
\end{aligned}
$$

Looking back to (29), the required occurrence probability $\operatorname{Pr}(|\mathcal{D S}|=L)$ as well as the capacity $C^{L}$ are available. This gives the following theorem:

Theorem 3: In the presence of outdated CSI, the end-to-end ergodic capacity for the proposed scheme over i.i.d. Rayleigh channels can be calculated in a closed form by (21).

Proof: Substituting (15), (32) and (34) into (29), yields (21).

\section{B. Capacity of GSC}

In the GSC scheme, the source broadcasts a signal and those relays who correctly decode this signal constitute a decoding subset $\mathcal{D S}$. $N$ relays with the largest SNRs in the relay-destination channels are selected from $\mathcal{D S}$. The regenerated signals are orthogonally transmitted from the selected relays to the destination. For example, all selected relays can retransmit via orthogonal frequency-division multiplexing access (OFDMA) [24]. Using maximal ratio combining at the receiver, the total received SNR is $\gamma_{t o t}=\sum_{n=1}^{N} \gamma_{\tilde{k}_{n}, d}$, which is identical to that of OSTC. It can be verified that the MGF of GSC is as same as that of OSTC and can also be expressed by (23). The key difference between two schemes is that GSC uses totally $N+1$ orthogonal channels for the end-to-end signal transmission, whereas the number of required channels is merely 2 in the proposed scheme thanks to the utilization of space-time coding.

Corollary 1: The outage probability of GSC scheme is identical to that of OSTC given in (20), i.e.,

$$
P_{g s c}\left(\gamma_{t h}\right)=P_{o s t c}\left(\gamma_{t h}\right) \text {. }
$$

The ergodic capacity of GSC can be obtained by replacing the factors $\beta_{L} /\left(1+\beta_{L}\right)$ and $\beta_{N} /\left(1+\beta_{N}\right)$ in $(21)$ by $1 /(L+1)$ and $1 /(N+1)$, respectively.

According to [24], the N+NT-ORS scheme can be transformed to GSC if a normalized threshold $u=1$ is used. For brevity, we only analyze GSC in this article since the mechanism and their achievable performance are quite similar.

\section{Capacity of ORS}

As mentioned in Section IV, the proposed OSTC scheme can be transformed into the conventional ORS if the number of selected relays is decreased to $N=1$. Accordingly, we can figure out ORS's capacity simply by setting $N=1$ in (21):

$$
C_{\text {ors }}=\left.C_{\text {ostc }}\right|_{N=1} \text {. }
$$

\section{NUMERICAL RESULTS}

We make use of Monte-Carlo simulations to validate analytical results of outage probability and ergodic capacity as well as the achievable diversity. Given i.i.d. Rayleigh channels with a normalized gain, a performance comparison of OSTC, ORS and GSC in the absence and presence of outdated CSI is carried out. The numerical results are obtained by iterating $10^{6}$ channel realizations, and an end-to-end target rate of $R=1 \mathrm{bps} / \mathrm{Hz}$ is applied for outage calculations. To guarantee a fairness comparison, the theoretical power mode defined in (1) is applied first. For brevity, the source's power is $P_{s}=0.5 P$, resulting in an average SNR $\bar{\gamma}_{s}=0.5 P / \sigma^{2}$ for the source-relay channels, while $\bar{\gamma}_{L}=0.5 P / L \sigma^{2}$ and $\bar{\gamma}_{N}=0.5 P / N \sigma^{2}$ for the relay-destination channels. In all figures, the numerical results are marked by markers, while the analytical results derived from (20) and (21) are plotted into curves. It can be seen that all markers strictly fall into their corresponding curves, which corroborate our theoretical analyses in this article.

We first investigate the impact of outdated CSI on a cooperative network with $K=8$ decode-and-forward relays, among which $N=2$ relays are selected for OSTC. As shown in Fig.2a, OSTC suffers from a little bit performance loss compared to ORS when the knowledge of CSI is prefect, i.e., $\rho=1$. That is because a single relay with the strongest SNR transmits the 


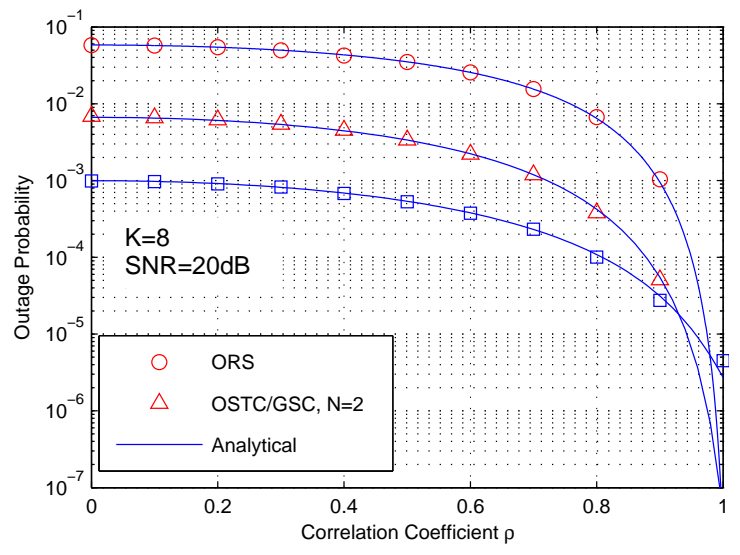

(a)

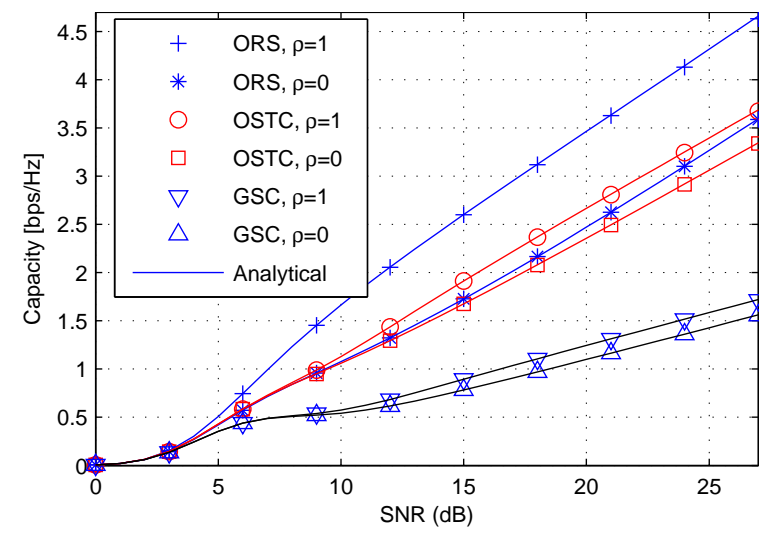

(b)

Fig. 3. (a) Outage probabilities of OSTC, ORS and GSC as a function of correlation coefficient $\rho \in[0,1]$ in the case of $K=8$ and $\bar{\gamma}=20 \mathrm{~dB}$; (b) A capacity comparison of OSTC, ORS and GSC as a function of the average SNR in the case of $K=8$ and $N=4$. Numerical results are marked by markers while analytical results by curves.

regenerated signal in ORS, while a pair of relays with the strongest and second strongest SNR are utilized in OSTC. In the case of $\rho=1$, the curve of OSTC is in parallel with its counterpart in ORS that has a full diversity of $K=8$. It can be therefore concluded that OSTC also achieves a diversity of $d=8$ and its outage probability decays at a rate of $1 / \bar{\gamma}^{8}$ in the high SNR. As a benchmark, we set up another cooperative network with $K=1$ and draw its performance curve of $\rho=1$, which has the diversity of $d=1$ and is indicated by $K=1, \rho=1$ in the legend. Similarly, $K=2, \rho=1$ denotes the curve of ORS achieving $d=2$. In the high SNR, OSTC's curves in the cases of $\rho=0$ and $\rho=0.5$ are both parallel with $K=2, \rho=1$, while the curves of ORS are parallel with $K=1, \rho=1$. That is to say, the diversity of ORS is 1 in the presence of outdated CSI, whereas an order of 2 is still kept by OSTC thanks to using $N=2$ selected relays.

As depicted in Theorem 2, the achievable diversity of OSTC equals to $N$ in the presence of outdated CSI, even if the outdated CSI tends to the actual CSI $(\rho \rightarrow 1)$, whereas a fulldiversity order of $K$ is available when the knowledge of CSI is prefect. To prove this, OSTC's outage probability in the high SNR (up to $50 \mathrm{~dB}$ ) is provided in Fig.2b. As a benchmark, the curves of $K=2, \rho=1$ and $K=8, \rho=1$ achieving $d=2$ and $d=8$, respectively, are given in this figure. As can be seen, OSTC's curves are all parallel with the curve of $K=2, \rho=1$ in the high SNR regardless of which correlation coefficients are used (e.g., $\rho=0.9,0.95$ and 0.99 ). Only the outage probability of $\rho=1$ decays at a different and faster rate. That is to say, the diversity of OSTC is $d=2$ when $\rho<1$ but the full diversity of $d=8$ when $\rho=1$. Hence, Theorem 2 is justified.

Analytical and numerical results of OSTC, ORS and GSC as a function of $\rho$ are given in Fig.3a. It can be observed that OSTC and GSC achieve an identical performance for any value of $\rho$. Given $K=8$ and $\bar{\gamma}=20 \mathrm{~dB}$, OSTC and GSC in the case of $N=2$ outperform ORS almost an order of magnitude, and nearly 2 orders of magnitude if the number of selected relays is increased to $N=4$. When the used CSI is prefect, OSTC suffers from a performance loss, but it is relatively small. Although the cooperative system becomes more reliable with an increased number of selected relays, there exists a capacity loss that is proportional to $N$, which will be shown as follows.

Fig. $3 b$ compares capacities of different schemes as a function of the average SNR $\bar{\gamma}$. The number of cooperating relays is assumed to be $K=8$ and the number of selected relays for OSTC and GSC is $N=4$. When the used CSI is perfect, OSTC suffers from a capacity loss compared to ORS since the applied 4 -dimensional OSTBC supports a maximal rate of only $3 / 4$ in relay-destination link. Due to the use of 4 orthogonal channels, equivalent to a rate of $1 / 4$, the loss of GSC is more severe. As an example, we can observe that $4.3 \mathrm{bps} / \mathrm{Hz}, 3.4 \mathrm{bps} / \mathrm{Hz}$ and $1.6 \mathrm{bps} / \mathrm{Hz}$ at a given SNR of $\bar{\gamma}=25 \mathrm{~dB}$ are achieved by ORS, OSTC and GSC, respectively. In the case of $\rho=0$, the capacity of OSTC approaches that of ORS with a loss of less than $0.2 \mathrm{bps} / \mathrm{Hz}$. That is to say, despite a diversity gain of $d=4$ achieved by OSTC in the presence of outdated CSI, the price on the capacity loss is negligible. In comparison, GSC's capacity is around $1.5 \mathrm{bps} / \mathrm{Hz}$ at a given $\mathrm{SNR}$ of $25 \mathrm{~dB}$, less than a half of ORS and OSTC. On the other hand, ORS is vulnerable to the outdated CSI because its capacity reduces from $4.3 \mathrm{bps} / \mathrm{Hz}$ to $3.3 \mathrm{bps} / \mathrm{Hz}$ when $\rho=1$ is changed to $\rho=0$, equivalent to a loss of $1 \mathrm{bps} / \mathrm{Hz}$. In contrast, the capacity loss is less than $0.3 \mathrm{bps} / \mathrm{Hz}$ for OSTC and GSC, implying their effectiveness of combatting the outdated CSI and their 'robustness' feature we pursued in this article.

A capacity comparison as a function of the number of selected relays is given in Fig.4a, where $K=16$ and $\bar{\gamma}=20 \mathrm{~dB}$ are applied. When $N=1$, all schemes achieve the same performance since both OSTC and GSC are transformed to ORS if a single relay is selected. If the number of selected relays is more than $N=5$, the capacity of OSTC become nearly constant and are around $1.9 \mathrm{bps} / \mathrm{Hz}$, accounting for approximate $75 \%$ of ORS's capacity. GSC's capacity continuously decreases with the increased number of $N$. Using $N=16$ as an example, its capacity is $0.4 \mathrm{bps} / \mathrm{Hz}$, accounting for only $16 \%$ of ORS and $21 \%$ of OSTC. That is to say, OSTC achieves 5-times higher capacity over GSC, although they obtain the same diversity in the presence of outdated CSI. On the other hand, ORS is 


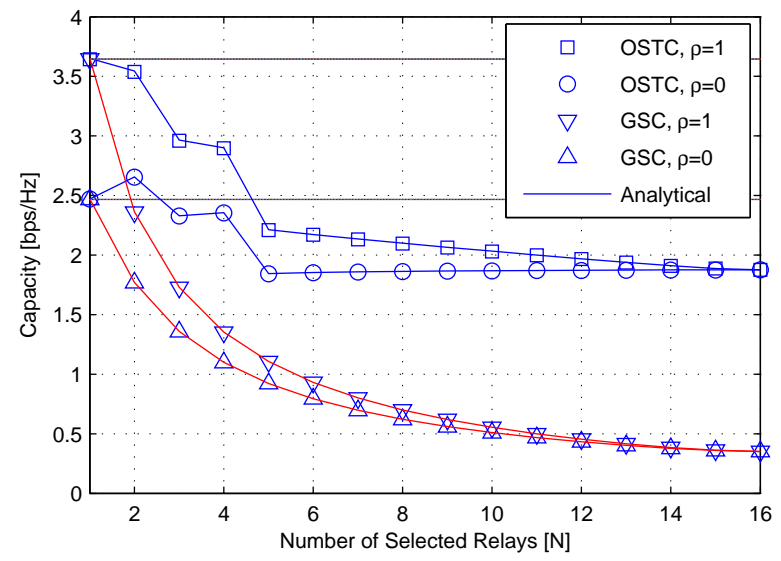

(a)

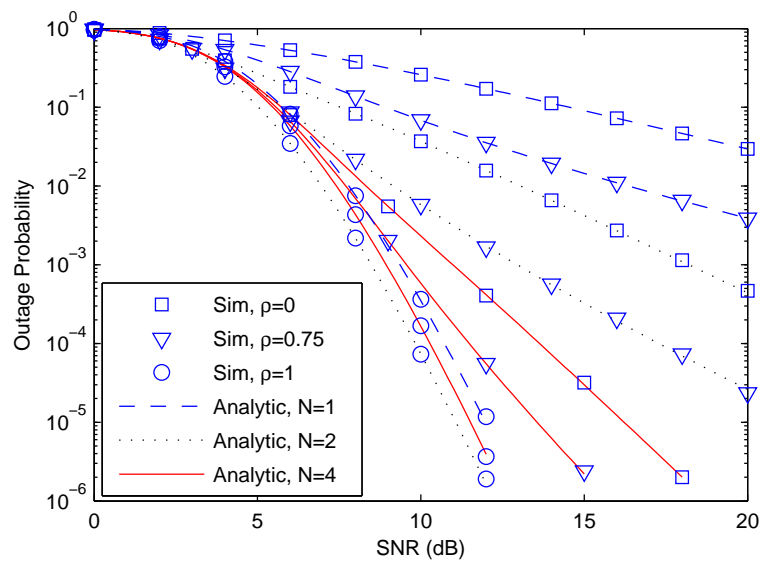

(b)

Fig. 4. (a) A capacity comparison of OSTC, ORS and GSC as a function of the number of selected relays $N \in[1,16]$ in the case of $K=16$ and $\bar{\gamma}=20 \mathrm{~dB}$; (b) Outage probabilities of OSTC and ORS as a function of the average SNR $P_{u} / \sigma^{2}$ for the practical power mode in the case of $K=10$. Numerical results are marked by markers while analytical results by curves.

vulnerable to the outdated CSI since its capacity reduces from 3.6 to $2.5 \mathrm{bps} / \mathrm{Hz}$ when $\rho=1$ is changed to $\rho=0$, equivalent to a loss of $1.1 \mathrm{bps} / \mathrm{Hz}$. In contrast, the capacity loss of OSTC and GSC are far smaller, e.g., less than $0.2 \mathrm{bps} / \mathrm{Hz}$ in the case of $N=8$, implying their effectiveness of combatting the outdated CSI and their 'robustness' feature we pursued.

In addition to the fair comparison under the same end-toend power constraint, the simulation taking advantage of the practical power mode depicted in (2) has also been carried out. Outage probabilities of OSTC and ORS as a function of the average SNR $P_{u} / \sigma^{2}$ in the case of $K=10$ are illustrated in Fig.4b. Contrary to the performance loss shown in Fig.2a, OSTC achieves a better performance than ORS in the case of $\rho=1$. That is because the transmit power of relay is fixed to $P_{u}$ and the number of selected relays for OSTC are $N=2$ and $N=4$, resulting in double and four-times power consumptions than ORS. Interestingly, the outage probability of $N=4$ is worse than that of $N=2$, although the former consumes more power. That is because the Alamouti scheme for $N=2$ is the unique full-rate full-diversity coding, while 4-dimensional OSTBC supports a maximal rate of only $3 / 4$. We can conclude that the proposed scheme outperforms ORS and GSC in the practical power mode, and the achieved performance gain is more remarkably than in the theoretical power mode.

\section{CONCLUSIONS}

In this article, we proposed a robust opportunistic relaying scheme to combat the outdated CSI for decode-and-forward cooperative systems. The proposed scheme opportunistically selects a predefined number of relays, rather than a single relay in the conventional ORS, to decode and simultaneously retransmit the original signal. When the knowledge of CSI is prefect, the full diversity of $K$, i.e., the number of cooperating relays, can be achieved. In the presence of outdated CSI, where the outage probability of ORS drastically deteriorates and its diversity degrades to one, i.e., no diversity, the diversity of $N$ can still be kept by this scheme. Compared with GSC, the capacity loss of this scheme is negligible thanks to the simultaneous transmission by means of space-time coding. In comparison with DSTC, a relay selection process is introduced so that the number of selected relays is fixed and known beforehand by the whole cooperative system, which makes sense for the practical systems. From the perspective of both the achieved performance and implementation complexity, the proposed scheme is the best solution until now. Next, the following works are worth being explored: 1) making clear the optimal number of selected relays from the viewpoint of multiplexing-diversity trade-off, 2) designing a robust scheme for amplify-and-forward opportunistic relaying, and 3) considering the multi-antenna system at the relay.

\section{ACKNOWLEDGMENT}

This work was conducted within the framework of the H2020 5G-PPP COHERENT project and FP7 ABSOLUTE project, which are funded by the Commission of the European Union. The authors would like to thank the editors, especially Prof. Wolfgang Gerstacker, for their great efforts during the review process. The insightful comments of the anonymous reviewers that helped improve the quality of this paper are gratefully appreciated.

\section{APPENDIX A}

\section{DERIVATION OF LEMMA 1}

Using the maximal ratio combining at the receiver, the total SNR of GSC is $\gamma_{t o t}=\sum_{n=1}^{N} \gamma_{\tilde{k}_{n}, d}$, which is identical to that of OSTC. Since the N+NT-ORS scheme can be transformed to GSC if the normalized threshold $u=1$ is used, according to Eq. (15) of [24], the MGF of OSTC/GSC can be expressed as

$$
\begin{aligned}
\mathcal{M}_{\gamma}(s) & =L\left(\begin{array}{c}
L-1 \\
N-1
\end{array}\right) \sum_{l=0}^{L-N} \sum_{t=0}^{L-N-l} \sum_{m=0}^{l}\left(\frac{1}{1+s \bar{\gamma}_{N}}\right)^{L-l-1} \\
& \times \frac{(-1)^{t+m}(L-N) !}{(L-N-l-t) ! m ! t !(l-m) !} \cdot \frac{1}{\eta(s)}
\end{aligned}
$$

where

$$
\eta(s)=\left(1+s \bar{\gamma}_{N}\right)(L-l)+m\left[1+s \bar{\gamma}_{N}\left(1-\rho^{2}\right)\right] .
$$


From [37], we can derive the following equality:

$$
\sum_{t=0}^{T} \frac{(-1)^{t}}{(T-t) ! t !}=\left\{\begin{array}{ll}
1, & T=0 \\
0, & T>0
\end{array} .\right.
$$

It can be known that the second and third sum of (35) denoted by $\phi(l, \rho)$ and $\psi(l)$ in the following equation, respectively, are independent with each other. Hence, we can rewrite (35) as

$$
\begin{aligned}
\mathcal{M}_{\gamma}(s) & =L(L-N) !\left(\begin{array}{c}
L-1 \\
N-1
\end{array}\right) \sum_{l=0}^{L-N}\left(\frac{1}{1+s \bar{\gamma}_{N}}\right)^{L-1-l} \\
& \times \underbrace{\sum_{m=0}^{l} \frac{(-1)^{m}}{m !(l-m) !} \cdot \frac{1}{\eta(s)}}_{\phi(l, \rho)} \\
& \times \underbrace{\sum_{t=0}^{L-N-l} \frac{(-1)^{t}}{(L-N-l-t) ! t !}}_{\psi(l)} .
\end{aligned}
$$

Replacing $T$ in the equality of (36) by $L-N-l$, we know that $\psi(l)$ is nonzero only when $L-N-l=0$, that is

$$
\psi(l)=\sum_{t=0}^{L-N-l} \frac{(-1)^{t}}{(L-N-l-t) ! t !}=\left\{\begin{array}{ll}
1, & l=L-N \\
0, & l \neq L-N
\end{array} .\right.
$$

That is to say, only the item in the case of $l=L-N$ is nonzero, while others are all equal to zero. Therefore, we expand (37) and remove the items of $l \neq L-N$, yielding (23) and (24).

\section{APPENDIX B}

\section{ASYMPTOTIC ANALYSIS OF THEOREM 2}

In the high SNR regime, the average SNRs $\bar{\gamma}_{s}, \bar{\gamma}_{L}$ and $\bar{\gamma}_{N}$ satisfy an assumption of $1 / \bar{\gamma}_{x} \rightarrow 0$. For simplicity, their subscripts are neglected and denoted hereinafter by a unified notation of $\bar{\gamma}$. As we can see in (20), OSTC's outage probability behaves in a different way with respect to the value of $\rho$, so our asymptotic analysis needs to be carried out upon three extreme cases:

1) $\rho=1$ : When the knowledge of CSI is prefect, the function given in (24) can be simplified into $\eta(s)=N(1+s \bar{\gamma})+m$. Then, the MGF given in (23) is rewritten as

$$
\begin{aligned}
\mathcal{M}_{\gamma}(s) & =L(L-N) !\left(\begin{array}{c}
L-1 \\
N-1
\end{array}\right)\left(\frac{1}{1+s \bar{\gamma}}\right)^{N-1} \\
& \times \underbrace{\sum_{m=0}^{L-N} \frac{(-1)^{m}}{m !(L-N-m) !} \cdot \frac{1}{N(1+s \bar{\gamma})+m}}_{\omega(s)} .
\end{aligned}
$$

Referring to [37], we have the following equality:

$$
\sum_{m=0}^{M} \frac{(-1)^{m}}{m !(M-m) !} \cdot \frac{1}{\alpha+m}=\prod_{m=0}^{M} \frac{1}{\alpha+m} .
$$

It is obvious that $\omega(s)$ in (38) is exactly a sample of this equality in the case of $M=L-N$ and $\alpha=N(1+s \bar{\gamma})$. Accordingly, $\omega(s)$ can be transformed to

$$
\omega(s)=\prod_{m=0}^{L-N}\left(\frac{1}{N(1+s \bar{\gamma})+m}\right) .
$$

Neglecting $m$ due to $\bar{\gamma} \gg m$ in high SNR, then

$$
\omega(s) \approx\left(\frac{1}{N(1+s \bar{\gamma})}\right)^{L-N+1}
$$

Substituting (39) into (38), yields

$$
\mathcal{M}_{\gamma}(s) \approx \frac{L(L-N) !}{N^{L-N+1}}\left(\begin{array}{c}
L-1 \\
N-1
\end{array}\right)\left(\frac{1}{1+s \bar{\gamma}}\right)^{L} .
$$

According to proposition 3 of [38], the diversity order equals to the pole of MGF. Letting $s \rightarrow \infty$, (40) can be rewritten as

$$
\mathcal{M}_{\gamma}(s) \approx\left(\frac{1}{s}\right)^{L}
$$

We get that the pole of (41) equals to $L$ and therefore its corresponding diversity is $L$. In terms of proposition 5 of [38], the asymptotic form of $\operatorname{Pr}($ outage $\mid L)$ in (25) is expressed as

$$
\operatorname{Pr}(\text { outage } \mid L)=\left(\frac{1}{\bar{\gamma}}\right)^{L}+o\left(\left(\frac{1}{\bar{\gamma}}\right)^{L}\right)
$$

where $o(x)$ is the little-o notation standing for a quantity that is ultimately smaller than $x$.

On the other hand, we can know that the diversity order of $\operatorname{Pr}($ outage $\mid L)$ is also $L$ from its closed-form expression given in (18). Similar to (42), its asymptotic form is given by

$$
\operatorname{Pr}(\text { outage } \mid L)=\left(\frac{1}{\bar{\gamma}}\right)^{L}+o\left(\left(\frac{1}{\bar{\gamma}}\right)^{L}\right) .
$$

Applying the Taylor series expansion centered at 0 , we have

$$
e^{x}=1+x+x^{2} / 2 !+\ldots
$$

Therefore, the occurrence probability in (15) can be expanded to the following asymptotic form:

$$
\operatorname{Pr}(|\mathcal{D S}|=L)=\left(\frac{1}{\bar{\gamma}}\right)^{K-L}+o\left(\left(\frac{1}{\bar{\gamma}}\right)^{K-L}\right) .
$$

Substituting (42), (43) and (44) into (13), we can get the asymptotic form of the overall outage probability as

$$
\begin{aligned}
P_{\text {ostc }}^{\prime} & =\left(\frac{1}{\bar{\gamma}}\right)^{K}+\sum_{L=1}^{N}\left(\left(\frac{1}{\bar{\gamma}}\right)^{K}+o\left(\left(\frac{1}{\bar{\gamma}}\right)^{K}\right)\right) \\
& +\sum_{L=N+1}^{K}\left(\left(\frac{1}{\bar{\gamma}}\right)^{K}+o\left(\left(\frac{1}{\bar{\gamma}}\right)^{K}\right)\right) \\
& =\left(\frac{1}{\bar{\gamma}}\right)^{K}+o\left(\left(\frac{1}{\bar{\gamma}}\right)^{K}\right) .
\end{aligned}
$$

According to the definition of diversity order [13], we have

$$
d=-\lim _{\bar{\gamma} \rightarrow \infty} \frac{\log \left(P_{\text {ostc }}^{\prime}\right)}{\log (\bar{\gamma})}=-\lim _{\bar{\gamma} \rightarrow \infty} \frac{-\log \bar{\gamma}^{K}}{\log \bar{\gamma}}=K .
$$


2) $\rho=0$ : If the actual CSI and its outdated version are independent of each other, (24) changes to $\eta(s)=(1+s \bar{\gamma})(N+m)$. Then, the MGF in (23) can be rewritten as

$$
\begin{aligned}
\mathcal{M}_{\gamma}(s) & =L(L-N) !\left(\begin{array}{c}
L-1 \\
N-1
\end{array}\right)\left(\frac{1}{1+s \bar{\gamma}}\right)^{N} \\
& \times \sum_{m=0}^{L-N} \frac{(-1)^{m}}{m !(L-N-m) !} \cdot \frac{1}{(N+m)} .
\end{aligned}
$$

Letting $s \rightarrow \infty$, it is further transformed to

$$
\mathcal{M}_{\gamma}(s)=\left(\frac{1}{s}\right)^{N}+o\left(\left(\frac{1}{s}\right)^{N}\right) .
$$

In accordance of proposition 3 and proposition 5 of [38], we know that the diversity order of $\operatorname{Pr}($ outage $\mid L)$ in (25) is $N$ and its asymptotic form can be given by

$$
\operatorname{Pr}(\text { outage } \mid L)=\left(\frac{1}{\bar{\gamma}}\right)^{N}+o\left(\left(\frac{1}{\bar{\gamma}}\right)^{N}\right) .
$$

Since (15) and (18) are independent of the correlation coefficient, their asymptotic forms are unchangeable for different values of $\rho$. Hence, (43) and (44) can be reused for this case. Substituting (43), (44) and (46) into (13), yields

$$
P_{\text {ostc }}^{\prime}=\left(\frac{1}{\bar{\gamma}}\right)^{N}+o\left(\left(\frac{1}{\bar{\gamma}}\right)^{N}\right) .
$$

Similar to (45), the achievable diversity for $\rho=0$ is

$$
d=-\lim _{\bar{\gamma} \rightarrow \infty} \frac{-\log \bar{\gamma}^{N}}{\log \bar{\gamma}}=N
$$

3) $\rho \rightarrow 1$ : As we know, one of the Taylor series expansion centered at 0 is

$$
\frac{1}{1-x}=\sum_{n=0}^{\infty} x^{n}
$$

The item $1 / \eta(s)$ in (23) can be expanded with respect to $1-\rho^{2} \rightarrow 0$, we have

$$
\begin{aligned}
\frac{1}{\eta(s)} & =\frac{1}{N(1+s \bar{\gamma})+m} \cdot \frac{1}{1+\frac{m s \bar{\gamma}\left(1-\rho^{2}\right)}{N(1+s \bar{\gamma})+m}} \\
& =\frac{1}{N(1+s \bar{\gamma})+m} \sum_{n=0}^{\infty}\left(\frac{-m s \bar{\gamma}\left(1-\rho^{2}\right)}{N(1+s \bar{\gamma})+m}\right)^{n} .
\end{aligned}
$$

If $s \rightarrow \infty$, substituting (48) into (23), we can get

$$
\mathcal{M}_{\gamma}(s)=\left(\frac{1}{s}\right)^{N}+o\left(\left(\frac{1}{s}\right)^{N}\right) .
$$

In accordance to proposition 3 and proposition 5 of [38], it is derived that the diversity order of $\operatorname{Pr}($ outage $\mid L)$ in (25) is $N$. Hence, its asymptotic form can also be expressed by (46). Similar to (47), we can get a diversity order of $d=N$ in the case of $\rho \rightarrow 1$.

According to [13], the outage probability is a decreasing function as $\rho$, we can get

$$
d_{\rho=0} \leqslant d_{\rho^{\prime}} \leqslant d_{\rho \rightarrow 1},
$$

where $\rho^{\prime}$ stands for an arbitrary value of $\rho \in(0,1)$. Since both the low and high end of $d$ is equal to $N$, i.e., $d_{\rho=0}=d_{\rho \rightarrow 1}=N$, it can be concluded that the diversity order of the proposed scheme is always $d=N$ when $\rho<1$.

\section{REFERENCES}

[1] D. Tse and P. Viswanath, Fundamentals of Wireless Communication. Cambridge, UK: Cambridge Univ. Press, 2005.

[2] J. Hoydis, S. ten Brink, and M. Debbah, "Massive MIMO in the UL/DL of cellular networks: How many antennas do we need?" IEEE J. Sel. Areas Commun., vol. 31, no. 2, pp. 160-171, Feb. 2013.

[3] Z. Pi and F. Khan, "An introduction to millimeter-wave mobile broadband systems," IEEE Commun. Mag., vol. 49, no. 6, pp. 101-107, Jun. 2011.

[4] A. Sendonaris, E. Erkip, and B. Aazhang, "User cooperation diversityPart I and II," IEEE Trans. Commun., vol. 51, no. 11, pp. 1927-1948, Nov. 2003.

[5] T. M. Cover and A. A. E. Gamal, "Capacity theorems for the relay channel," IEEE Trans. Inf. Theory, vol. 25, no. 5, pp. 572-584, Sep. 1979.

[6] J. N. Laneman, D. Tse, and G. W. Wornell, "Cooperative diversity in wireless networks: efficient protocols and outage behaviour," IEEE Trans. Inf. Theory, vol. 50, no. 12, pp. 3062-3080, Dec. 2004.

[7] M. Torabi, D. Haccoun, and J.-F. Frigon, "Relay selection in AF cooperative systems: An overview," IEEE Veh. Technol. Mag., vol. 7, no. 4, pp. 104-113, Dec. 2012.

[8] L. Xiao and X. Dong, "Unified analysis of generalized selection combining with normalized threshold test per branch," IEEE Trans. Wireless Commun., vol. 5, no. 8, pp. 2153-2163, Aug. 2006.

[9] Y. Jing and H. Jafarkhani, "Network beamforming using relays with perfect channel information," IEEE Trans. Inf. Theory, vol. 55, no. 6 , pp. 2499-2517, Jun. 2009.

[10] J. N. Laneman and G. W. Wornell, "Distributed space-time-coded protocols for exploiting cooperative diversity in wireless networks," IEEE Trans. Inf. Theory, vol. 49, no. 10, pp. 2415-2425, Oct. 2003.

[11] A. Bletsas et al., "A simple cooperative diversity method based on network path selection," IEEE J. Sel. Areas Commun., vol. 24, no. 3, pp. 659-672, Mar. 2006.

[12] A. Bletsas, H. Shin, and M. Z. Win, "Cooperative communications with outage-optimal opportunistic relaying," IEEE Trans. Wireless Commun., vol. 6, no. 9, pp. 3450-3460, Sep. 2007.

[13] J. L. Vicario et al., "Opportunistic relay selection with outdated CSI: Outage probability and diversity analysis," IEEE Trans. Wireless Commun., vol. 8, no. 6, pp. 2872-2876, Jun. 2009.

[14] M. Seyfi et al., "Effect of feedback delay on the performance of cooperative networks with relay selection," IEEE Trans. Wireless Commun., vol. 10 , no. 12 , pp. 4161-4171, Dec. 2011.

[15] S. Kim, S. Park, and D. Hong, "Performance analysis of opportunistic relaying scheme with outdated channel information," IEEE Trans. Wireless Commun., vol. 12, no. 2, pp. 538-549, Feb. 2013.

[16] M. Torabi, D. Haccoun, and J.-F. Frigon, "Impact of outdated relay selection on the capacity of AF opportunistic relaying systems with adaptive transmission over non-identically distributed links," IEEE Trans. Wireless Commun., vol. 10, no. 11, pp. 3626-3631, Nov. 2011.

[17] M. Torabi and D. Haccoun, "Capacity of amplify-and-forward selective relaying with adaptive transmission under outdated channel information," IEEE Trans. Veh. Technol., vol. 60, no. 5, pp. 2416-2422, Jun. 2011.

[18] — "Capacity analysis of opportunistic relaying in cooperative systems with outdated channel information," IEEE Commun. Lett., vol. 14, no. 12, pp. 1137-1139, Dec. 2010.

[19] N. S. Ferdinand, N. Rajatheva, and M. Latva-aho, "Effects of feedback delay in partial relay selection over Nakagami-m fading channels," IEEE Trans. Veh. Technol., vol. 61, no. 4, pp. 1620-1634, May 2012.

[20] M. Soysa et al., "Partial and opportunistic relay selection with outdated channel estimates," IEEE Trans. Commun., vol. 60, no. 3, pp. 840-850, Mar. 2012.

[21] O. Amin, S. S. Ikki, and M. Uysal, "On the performance analysis of multirelay cooperative diversity systems with channel estimation errors," IEEE Trans. Veh. Technol., vol. 60, no. 5, pp. 2050-2059, Jun. 2011.

[22] B. Zhao and M. C. Valenti, "Practical relay networks: A generalization of hybrid-ARQ," IEEE J. Sel. Areas Commun., vol. 23, no. 1, pp. 7-18, Jan. 2005

[23] Y. Li et al., "On the design of relay selection strategies in regenerative cooperative networks with outdated CSI," IEEE Trans. Wireless Commun., vol. 10, no. 9, pp. 3086-3097, Sep. 2011.

[24] M. Chen, T. C.-K. Liu, and X. Dong, "Opportunistic multiple relay selection with outdated channel state information," IEEE Trans. Veh. Technol., vol. 61, no. 3, pp. 1333-1345, Mar. 2012. 
[25] V. Tarokh, H. Jafarkhani, and A. R. Calderbank, "Space-time block codes from orthogonal designs," IEEE Trans. Inf. Theory, vol. 45, no. 5, pp. 1456-1467, Jul. 1999.

[26] W. Jiang, H. Cao, and T. Kaiser, "Opportunistic space-time coding to exploit cooperative diversity in fast-fading channels," in Proc. IEEE ICC'2014, Sydney, Australia, Jun. 2014, pp. 4814-4819.

[27] A. A. Nasir et al., "Timing and carrier synchronization with channel estimation in multi-relay cooperative networks," IEEE Trans. Signal Process., vol. 60, no. 2, pp. 793-811, Feb. 2012.

[28] H. Mehrpouyan et al., "Bounds and algorithms for multiple frequency offset estimation in cooperative networks," IEEE Trans. Wireless Commun., vol. 10, no. 4, pp. 1300-1311, Apr. 2011.

[29] W. Jiang, H. Cao, and T. Kaiser, "Power optimal allocation in decodeand-forward opportunistic relaying," in Proc. IEEE WCNC'2014, Istanbul, Turkey, Apr. 2014, pp. 1001-1006.

[30] T. R. Ramya and S. Bhashyam, "Using delayed feedback for antenna selection in MIMO systems," IEEE Trans. Wireless Commun., vol. 8, no. 12 , pp. 6059-6067, Dec. 2009.

[31] W. Jiang, H. Cao, and T. Kaiser, "An MGF-based performance analysis of opportunistic relay selection with outdated CSI," in Proc. IEEE VTC'2014-Spring, Seoul, South Korea, May 2014.

[32] S. M. Alamouti, "A simple transmit diversity technique for wireless communications," IEEE J. Sel. Areas Commun., vol. 16, no. 8, pp. 14511458, Oct. 1998.

[33] Y. Zhao, R. Adve, and T. J. Lim, "Improving amplify-and-forward relay networks: Optimal power allocation versus selection," IEEE Trans. Wireless Commun., vol. 6, no. 8, pp. 3114-3123, Aug. 2007.

[34] O. Ibe, Fundamentals of Applied Probability and Random Processes. Elsevier Science, 2005, p. 137.

[35] Y.-C. Ko, M.-S. Alouini, and M. K. Simon, "Outage probability of diversity systems over generalized fading channels," IEEE Trans. Commun., vol. 48, no. 11, pp. 1783-1787, Nov. 2000.

[36] F. Yilmaz and M.-S. Alouini, "A unified MGF-based capacity analysis of diversity combiners over generalized fading channels," IEEE Trans. Commun., vol. 60, no. 3, pp. 862-875, Mar. 2012.

[37] I. Gradsthteyn and I. Ryzhik, Table of Integrals, Series and Products. London, UK: Elsevier Inc., 2007.

[38] Z. Wang and G. B. Giannakis, "A simple and general parameterization quantifying performance in fading channels," IEEE Trans. Commun., vol. 51, no. 8, pp. 1389-1398, Aug. 2003.

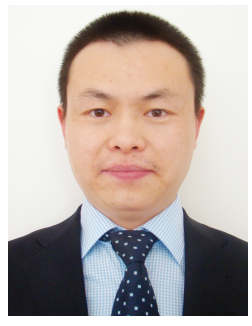

Wei Jiang (M'09) received his Ph.D degree from Beijing University of Posts and Telecommunications (BUPT) in 2008. Since Mar. 2008, he has been worked more than 4 years in Central Research Institute of Huawei Technologies, in the field of wireless communications and 3GPP standardization. In Sept. 2012, he joined the institute of digital signal processing (DSV), University of Duisburg-Essen, Germany, where he was a Postdoctoral researcher and worked for EU FP7 ABSOLUTE project and H2020 5G-PPP COHERENT project. Since Dec. 2015, he joined the Intelligent Networking group, German Research Center for Artificial Intelligence (DFKI) as a senior researcher and works for H2020 5GPPP SELFNET project. At the same time, he also works for the Technische University (TU) Kaiserslautern as a Lecture. He served as a vice Chair of IEEE TCCN special interest group (SIG) "Cognitive Radio in $5 G$ ". He is the author of more than 30 papers in top international journals and conference proceedings, and has 27 patent applications in wireless communications, most of which have already been authorized in China, Europe, United States or Japan. He wrote a chapter "From OFDM to FBMC: Principles and Comparisons" for the book "Signal Processing for 5G: Algorithms and Implementations" (Wiley, 2016). His present research interests are in digital signal processing, multi-antenna technology, and cooperative communications.

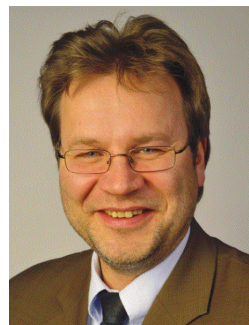

Thomas Kaiser (M'98-SM'04) received the Diploma degree in electrical engineering from RuhrUniversity Bochum, Bochum, Germany, in 1991 and the Ph.D. (with distinction) and German Habilitation degrees in electrical engineering from Gerhard Mercator University, Duisburg, Germany in 1995 and 2000, respectively. From 1995 to 1996, he spent a research leave with the University of Southern California, Los Angeles, which was grant-aided by the German Academic Exchange Service. From April 2000 to March 2001, he was the Head of the Department of Communication Systems, Gerhard Mercator University, and from April 2001 to March 2002, he was the Head of the Department of Wireless Chips and Systems, Fraunhofer Institute of Microelectronic Circuits and Systems, Duisburg. From April 2002 to July 2006, he was Coleader of the Smart Antenna Research Team, University of Duisburg-Essen, Duisburg. In the summer of 2005, he joined the Smart Antenna Research Group, Stanford University, Stanford, CA, and in the winter of 2007, he joined the Department of Electrical Engineering, Princeton University, Princeton, NJ, both as a Visiting Professor. From 2006 to 2011, he headed the Institute of Communication Technology at Leibniz University of Hannover, Germany. Currently, he heads the Institute of Digital Signal Processing at the University of Duisburg-Essen and is founder and CEO of ID4us GmbH, an RFIDcentric company. He is the author and co-author of more than 250 papers in international journals and conference proceedings and two books entitled Ultra Wideband Systems With MIMO (Wiley, 2010) and Digital Signal Processing for Passive RFID (Wiley, 2015). Dr. Kaiser was the founding Editor-inChief of the e-letter of the IEEE Signal Processing Society and the General Chair of the IEEE International Conference on UltraWideBand in 2008, the International Conference on Cognitive Radio Oriented Wireless Networks and Communications in 2009 and the IEEE Workshop on Cellular Cognitive Systems in 2014.

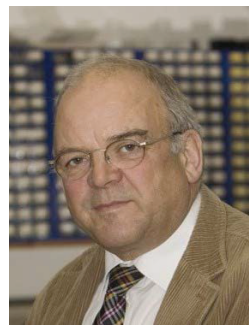

A. J. Han Vinck (M'77-SM'91-F'06) is a senior professor in Digital Communications at the University of Duisburg-Essen, Germany, since September 2014 and a member of the Institute of Digital Signal Processing at the Electrical Engineering Department. $\mathrm{He}$ is an adjunct professor at the National Cheng Kung University Tainan, Taiwan and honorary professor at the University of Johannesburg, South Africa. He was in the Institute for Experimental Mathematics at the University of Duisburg-Essen, Germany, since 1990, specializing in Information and Communication theory, Coding and Network aspects in digital communications. Professor Vinck held positions of professional responsibility including the Director of the Institute for Experimental Mathematics in Essen, founding Chairman of the IEEE German Information Theory chapter, President of the IEEE Information theory Society (2003) and President of the Leibniz foundation for the stimulation of research in the field of Information theory, Biology and Neuroscience. He received a number of accolades including the election by the IEEE as Fellow for his "Contributions to Coding Techniques", selected as Distinguished Lecturer for the Communications Society of the IEEE, the IEEE ISPLC 2006 Achievement award for contributions to Power Line Communications, and the SAIEE annual award for the best paper published in the SAIEE Africa Research Journal in 2008. He was awarded the 2015 Aaron D. Wyner Distinguished Service Award for outstanding leadership in, and provided long-standing, exceptional service to, the Information Theory community. He is instrumental in the organization of research forums including the IEEE Information Theory workshops (Veldhoven, 1990) and symposia (ISIT 1997, Ulm) Japan-Benelux workshops on Information theory (now Asia-Europe workshop on "Concepts in Information Theory") and the International Winter school on Coding and Information theory in Europe. He is author of the book Coding Concepts and Reed-Solomon Codes". 